\title{
Cyclophosphamide combined with mitoxantrone and cytarabine is an effective salvage regimen for patients with acute myeloid leukemia who experienced primary induction failure or relapse
}

\author{
SHUNING WEI, YINGCHANG MI, HUI WEI, DONG LIN, KANQI LIU, BENFA GONG, GUANGJI ZHANG, \\ YUNTAO LIU, YAN LI, CHUNLIN ZHOU, BINGCHENG LIU, WEI LI and JIANXIANG WANG \\ Institute of Hematology and Blood Diseases Hospital, Chinese Academy \\ of Medical Sciences and Peking Union Medical College, Tianjin 300020, P.R. China
}

Received September 4, 2015; Accepted November 2, 2015

DOI: $10.3892 / \mathrm{mco} .2015 .679$

\begin{abstract}
Salvage regimens containing mitoxantrone have been successfully used for the treatment of primary induction failure or relapsed acute myeloid leukemia. However, the combination of cyclophosphamide with mitoxantrone and cytarabine (MAC) has never been assessed in these patients. A total of 91 patients, including patients with primary induction failure $(n=44)$ and relapsed $(n=47)$, were treated with a salvage MAC regimen, which consisted of mitoxantrone, cytarabine and cyclophosphamide. The overall complete remission rate was $74.7(68 / 91)$ and $72.7 \%$ (32/44) for primary induction failure, and $76.6 \%$ (36/47) for relapsed patients. The overall survival rate and disease-free survival rate were 72.1 and $59.7 \%$ at 1 year, 42.9 and $47.1 \%$ at 3 years, and 36.7 and $43.0 \%$ at 5 years, respectively. Only one patient succumbed to mortality during induction therapy. The toxicity level was acceptable. Therefore, the present study demonstrated that MAC is a highly effective and well-tolerated regimen for salvage in patients experiencing primary induction failure or relapse. This treatment significantly improved the prognosis.
\end{abstract}

\section{Introduction}

Considering patients with acute myeloid leukemia (AML), $>20 \%$ fail to achieve complete remission (CR) following a standard first line induction treatment (1). The majority of patients with CR relapsed within 1-2 years and the chance of long-term disease-free survival for relapsed patients is $<10 \%$ (2). For the majority of relapsed patients, regimens involving fludarabine

Correspondence to: Dr Jianxiang Wang, Institute of Hematology and Blood Diseases Hospital, Chinese Academy of Medical Sciences and Peking Union Medical College, 288 Nanjing Road, Tianjin 300020, P.R. China

E-mail: wangjx@ihcams.ac.cn

Key words: MAC regimen, primary induction failure, relapse, acute myeloid leukemia have been performed in previous studies for treatment, with a CR rate ranging between 30 and 60\% (3-8). Liso et al (9) and Kern et al (10) have also reported a high-dose of cytarabine, combined with mitoxantrone and etoposide, as a salvage regimen. In these cases, the CR rates were 47.4 and $47 \%$, and the median overall survival (OS) rates were 8.1 months and 4.2 months, respectively.

An optimum strategy for patients with primary induction failure or relapse remains to be elucidated. A pilot study was performed in order to assess the effect of the MAC regimen for re-induction therapy in patients who suffered primary induction failure or relapsed in the Institute of Hematology and Blood Diseases Hospital (Tianjin, China) . Cyclophosphamide, which is seldom used in the treatment of AML, was combined in this regimen. In the present report, the efficacy and toxicity of the MAC salvage regimen were assessed. The MAC salvage regimen was selected in the present study to reduce the leukemic burden and induce remission. It was hypothesized that there would be a longer survival rate as a result of this combination chemotherapy.

\section{Patients and methods}

Patients. Between September 2001 and October 2011,91 newly diagnosed patients with de novo AML in the Institute of Hematology and Blood Diseases Hospital (Tianjin, China) were enrolled in the present study. Patients with acute promyelocytic leukemia were excluded. Absence of heart, lung, liver, or kidney damage, as well as no active infection, was required for enrollment in the study. Written informed consent was obtained from each patient. The diagnosis was based on bone marrow cell morphology, cytochemistry, immunophenotyping and chromosomal analysis. The final follow-up was March 2014. For the entire cohort, the median follow-up time was 46 months.

Disease evaluation. The diagnosis and classification of AML were based on the criteria of the French-American-British (FAB) Cooperative Study Group (11) and the more recent World Health Organization classification (12). The disease status was assessed by bone-marrow cytology and flow cytometric 
analysis (FACSCalibur; Becton Dickinson, San Jose, CA, USA). Bone marrow or peripheral samples were harvested following 1-3 days of culture, containing no stimulating factors. Chromosomal analysis was performed using the R-banding method and karyotyped, according to the International System for Human Cytogenetic Nomenclature (13). Mutations in several genes, including NPM1, FLT3/ITD, FLT3/TKD, AML1, CEBPA, C-KIT and JAK2, were investigated. For cytogenetic analysis, the chromosome karyotype and/or fusion gene (e.g. AML1/ETO or CBF $\beta /$ MYH11) mutation was analyzed successfully in $85 / 87$ patients. A total of 85 patients were divided into three cytogenetic risk groups, according to the Southwest Oncology Group (SWOG) criteria: Favorable group with 28 cases, including $\mathrm{t}(8 ; 21)$ and inv(16); intermediate group with 45 cases, including normal karyotype, 2Y, 18, 16, del(12p); adverse group with 12 cases, including 25/27, abnormal 3q, 9q, 11q, 21q, 17p, t(6;9) and complex karyotype. Since the prognosis of the unknown group was similar to that of the intermediate group, these patients were incorporated into the intermediate group.

Treatment protocol. The initial induction regimen used in patients with primary induction failure $(n=44)$ included cytarabine (100 mg/m $/ \mathrm{m}^{2}$ infusion) for 7 consecutive days and daunorubicin $\left(45 \mathrm{mg} / \mathrm{m}^{2}\right)$ for 3 consecutive days, cytarabine (100 $\mathrm{mg} / \mathrm{m}^{2}$ infusion) for 7 consecutive days and Idamycin $\left(12 \mathrm{mg} / \mathrm{m}^{2}\right)$ for 3 consecutive days, and homoharringtonine (2-2.5 $\mathrm{mg} / \mathrm{m}^{2}$ infusion) for 7 consecutive days, a standard dose (sd) of cytarabine $\left(100 \mathrm{mg} / \mathrm{m}^{2}\right)$ for 7 consecutive days and daunorubicin $\left(45 \mathrm{mg} / \mathrm{m}^{2}\right)$ for 3 consecutive days. Daunorubicin or idarubicin, combined with $2-2.5 \mathrm{mg} \mathrm{m}^{-2} \cdot \mathrm{d}^{-1}$ homoharringtonine on days 1-7 and a sd or intermediate dose of cytarabine $\left(1 \mathrm{~g} \mathrm{~m}^{-2} \mathrm{q} 12 \mathrm{~h}\right.$ on days 5-7) was also performed as a first induction regimen.

The MAC regimen consisted of $8 \mathrm{mg} \mathrm{m}^{-2} \cdot \mathrm{d}^{-1}$ mitoxantrone on days $1-3,100 \mathrm{mg} \mathrm{m}{ }^{-2} \cdot \mathrm{d}^{-1}$ cytarabine on days 1-7 and $400 \mathrm{mg} \mathrm{m} \mathrm{m}^{-2} \cdot \mathrm{d}^{-1}$ cyclophosphamide on days 2 and 5 .

Efficacy and safety evaluations. The efficacy was established, according to the criteria of Cheson et al (14): i) CR, $\leq 5 \%$ blasts infiltration in bone marrow without Auer's rodes, with ANC $>1 \times 10^{9} / 1$ and PLT $>100 \times 10^{9} / 1$; ii) Partial remission, $5-25 \%$ of blast infiltration and blast reduction by $50 \%$ in bone marrow or $<5 \%$ of blasts in the bone marrow, however, with Auer's rods, and with a CR criteria for peripheral blood; iii) Non-remission, the patients who succumbed to mortality prior to the assessment of remission or those who failed to achieve CR or PR; iv) Early mortality was defined as mortality from any cause during the first 30 days from the beginning of the induction treatment. Patients were considered relapsed when they had $>5 \%$ blasts in the bone marrow examination.

Statistics. Disease-free survival (DFS) was calculated from the date of $\mathrm{CR}$ to the date of relapse, mortality, undergoing stem cells transplantation (SCT) or the final follow-up. The OS was calculated from the first day of MAC administration until mortality, undergoing SCT or the final follow-up.

Survival distributions were estimated by the Kaplan-Meier method. Statistical differences in survival distributions were evaluated using the log-rank test. Data analysis was performed using R 2.10.1 software (R Foundation for Statistical Computing, Vienna, Austria). $\mathrm{P}<0.05$ was considered to indicate a statistically significant difference.

\section{Results}

Patients. Of the 91 patients, 3 patients had a history of myelodysplastic syndrome. No patient had other hematological disorders at diagnosis. The median hemoglobin was $78.0 \mathrm{~g} / \mathrm{l}(36.0-136.0 \mathrm{~g} / \mathrm{l})$, the median white blood cell and platelet count was $29.5 \times 10^{9} / 1\left(1.1-417.8 \times 10^{9} / 1\right)$ and $36.0 \times 10^{9} / 1$ (4.0-305.0x $\left.10^{9} / 1\right)$, respectively. A total of 15 patients exhibited hyperleucocytosis (white blood cells $>100 \times 10^{9} / 1$ ). The blast count in bone marrow aspiration at diagnosis ranged between 28.5 and $97.0 \%$, the median blast count was $68.5 \%$. The clinical and hematological characteristics of these patients are listed in Table I. It was observed that 10/73 (13.7\%) patients exhibited FLT3/ITD, and FLT3/TKD was positive in another 2 patients. Karyotypic studies revealed unfavorable chromosomal abnormalities in 18 patients, including -7,3q, 11q and complex karyotype. A total of 11 and 53 patients were in favorable and intermediate groups, respectively. The other 9 patients exhibited no assessed cytogenetic factors. The risk categories were defined according to the SWOG criteria. A total of 44 patients experienced primary induction failure and 47 cases experienced relapse, including 20 cases of early relapse (42.6\%) and 27 cases of late relapse (57.4\%).

Response to MAC regimen. All 91 patients were assessed until the final follow-up and 68/91 (74.7\%) patients achieved $\mathrm{CR}(\mathrm{CR}+\mathrm{CR}$ with incomplete platelet recovery) following the MAC regimen. The CR rate was 72.7 (32/44) and 76.6\% (36/47) in patients with primary induction failure and those who experienced relapse, respectively. For the relapsed cases, it was $65.0(13 / 20)$ and $85.2 \%(23 / 27)$ in cases experienced early relapse and late relapse, respectively. No difference in the CR rate was observed between these groups. A total of 4/10 patients with FLT3/ITD positive achieved CR following the MAC regimen, PR and non-remission (NR) was observed in three cases, respectively. The overall CR rate was $81.8(9 / 11)$ and $79.2 \%$ (42/53) for patients with favorable and intermediate cytogenetic groups, while it was $66.7 \%$ $(12 / 18)$ for the unfavorable cytogenetic group. No difference in CR rate was observed for patients in different cytogenetic risk groups. The patients with unavailable cytogenetic factors had a CR rate of $55.5 \%$ (5/9). Early mortality was observed in only one case during the agranulocytosis period following chemotherapy, as a result of a severe lung infection and gastrointestinal hemorrhage.

A total of 27 patients underwent at least two high-dose cytarabine (Ara-c; $3 \mathrm{gm}^{-2} \mathrm{q} 12 \mathrm{~h}$ on days 1-3) or intermediate dose Ara-c plus anthracyclines prior to the MAC regimen. A total of 7/27 patients underwent SCT following the MAC salvage regimen.

Post-re-induction treatment. Among the 68 patients who achieved $\mathrm{CR}$ following the MAC regimen, five underwent allogenic-SCT, one succumbed to relapse following SCT, and four remain in CR. The other 63 patients entered consolidation 
Table I. Clinical and laboratory patient characteristics.

\begin{tabular}{lcc}
\hline Characteristic & No. patients $(\mathrm{n}=91)$ & $\%$ \\
\hline Median age, years & $37(14-72)$ & \\
Gender & & \\
Male & 47 & 51.6 \\
Female & 44 & 48.4 \\
FAB criteria & & \\
M0 & 2 & 2.2 \\
M1 & 4 & 4.4 \\
M2 & 31 & 34.1 \\
M4 (M4Eo) & $12(7)$ & $13.2(7.7)$ \\
M5 & 38 & 41.7 \\
M6 & 4 & 4.4 \\
Cytogenetic subgroups & & \\
Good & 11 & 12.1 \\
Intermediate & 53 & 58.2 \\
Poor & 18 & 19.8 \\
Not available & 9 & 9.9 \\
NCCN categories & & \\
Favorable & 9 & 21.9 \\
Intermediate & 39 & 29.9 \\
Unfavorable & 17 & \\
Not available & 26 & \\
Primary induction failure & 44 & \\
Early relapse & 20.9 \\
Late relapse & & \\
\hline
\end{tabular}

NCCN, national comprehensive cancer network; FAB, French-American-British; M4Eo, acute myelomonocytic leukemia with abnormal eosinophils.

chemotherapy. Among them, 26 patients were referred to 1-3 courses high-dose Ara-c ( $3 \mathrm{gm}^{-2} \mathrm{q} 12 \mathrm{~h}$ on days 1-3) or intermediate dose Ara-c plus anthracyclines consolidation chemotherapy, other patients received consolidation chemotherapy with daunorubicin, homoharringtonine, mitozantrone or amsacrine combined with a sd of cytarabine. A total of 32 patients remain alive in CR, 2/31 patients with relapse succumbed to subsequent progressive disease, and 2 patients with partial remission following the MAC regimen achieved $\mathrm{CR}$ with a sd of mitoxantrone and cytarabine. Each patient underwent allogenic-SCT and remain in CR. Among the 21 patients with NR, 18 failed to achieve CR even though they underwent G-CSF (300 $\mu \mathrm{g})$ administered 12 hours prior to fludarabine $\left(30 \mathrm{mg} / \mathrm{m}^{2}\right)$ in $0.5 \mathrm{~h}$ infusions for 5 consecutive days and cytarabine $\left(2 \mathrm{~g} / \mathrm{m}^{2}\right)$ in $4 \mathrm{~h}$ infusions for 5 consecutive days (FLAG), aclacinomycin $\left(5-7 \mathrm{mg} / \mathrm{m}^{2}\right)$ for 8 consecutive days, cytarabine $\left(10 \mathrm{mg} / \mathrm{m}^{2}\right)$ for 14 consecutive days and $\mathrm{G}-\mathrm{CSF}(300 \mu \mathrm{g})$ regimen re-induction and the final three were lost at follow-up.

OS and DFS. The median follow-up duration was 46 months (range, 2-69 months). The median OS of the 91 patients was 22 months (range, 0.5-73 months; Fig. 1). The

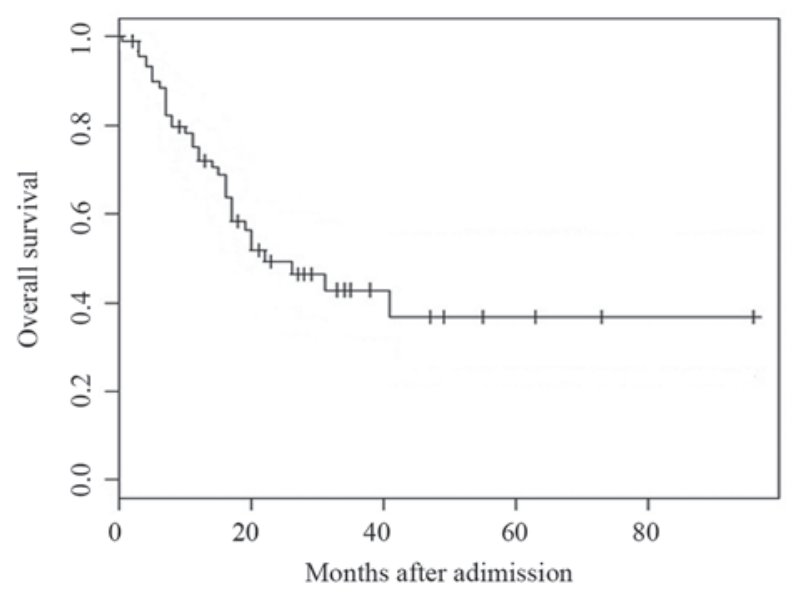

Figure 1. Overall survival for the entire study population. The overall survival at 1, 3 and 5 years for the entire study population were $72.1,42.9$ and $36.7 \%$, respectively.

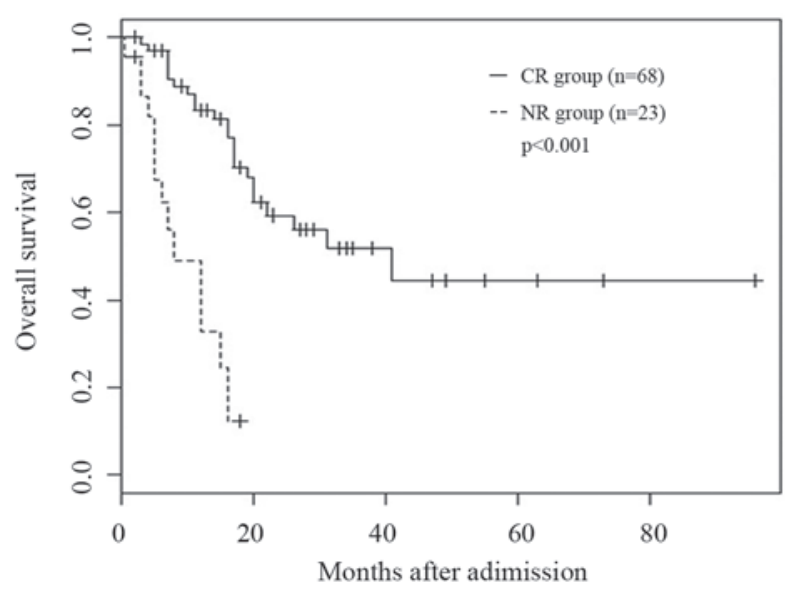

Figure 2. Overall survival for the CR and NR groups. For patients with CR $(\mathrm{n}=68)$ or not $(\mathrm{n}=23), 1$ and 3 year overall survival rates were 83.3 , vs. $32.7 \%$ $(\mathrm{P}<0.001)$ and 57.3 , vs. $10.3 \%$, respectively. CR, complete remission; NR, non-remission.

OS at 1, 3 and 5-years for the entire study population was $72.1,42.9$ and $36.7 \%$, respectively (Fig. 1).

For patients who attained CR $(n=68)$ or failed to $(\mathrm{n}=23), 1$ and 3 -year OS rate were 83.3, vs. 32.7\% $(\mathrm{P}<0.001)$ and 57.3, vs. $10.3 \%(\mathrm{P}<0.001)$, respectively (Fig. 2). The median survival was 12 (4-73) and 5 (0.5-20) months in patients with a white blood cell count $<100 \times 10^{9} / 1$ and $\geq 100 \times 10^{9} / 1(\mathrm{P}=0.0001)$, and it was 7.5 and 4 months in FLT3 positive and negative patients, respectively $(\mathrm{P}=0.0001)$. In a multivariate analysis, the white blood cell counts at diagnosis and FLT3 mutation were independent factors for $\mathrm{OS}(\mathrm{P}=0.035$ and $\mathrm{P}=0.008)$.

For the entire cohort, the 1 and 3 -year DFS rates were 48.1 and $38.0 \%$, respectively. The median DFS was 10 months (Fig. 3). For patients who attained CR following the MAC regimen, the DFS rate at 1,3 and 5-years was 59.7, 47.1 and 43\%, respectively (Fig. 4). For the OS rate $(\mathrm{P}=0.32)$, as well as the DFS rate $(\mathrm{P}=0.19)$, no difference was observed in different cytogenetic risk groups.

The median OS and DFS in seven transplanted patients was not significantly different compared with the group 


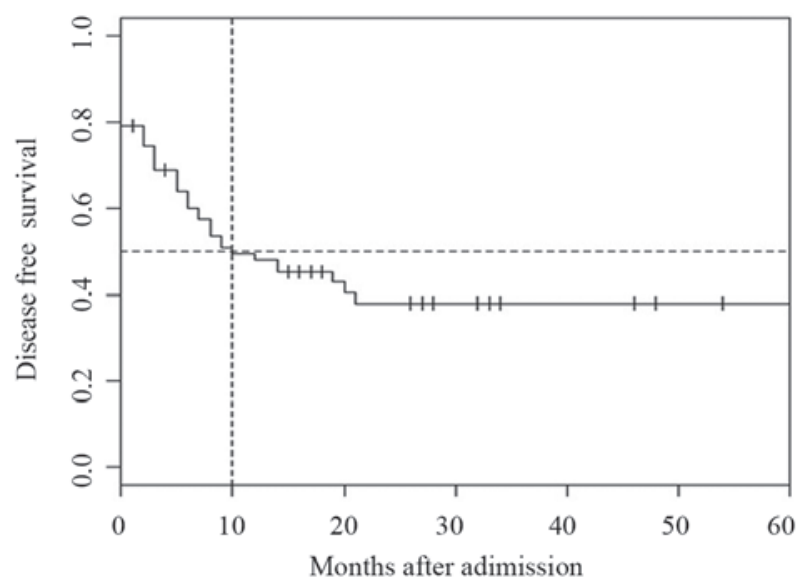

Figure 3. Disease free survival for the entire study population. For the entire cohort, the 1 and 3 year disease-free survival rates were 48.1 , vs. $38.0 \%$ respectively. The median disease-free survival rate was 10 months.

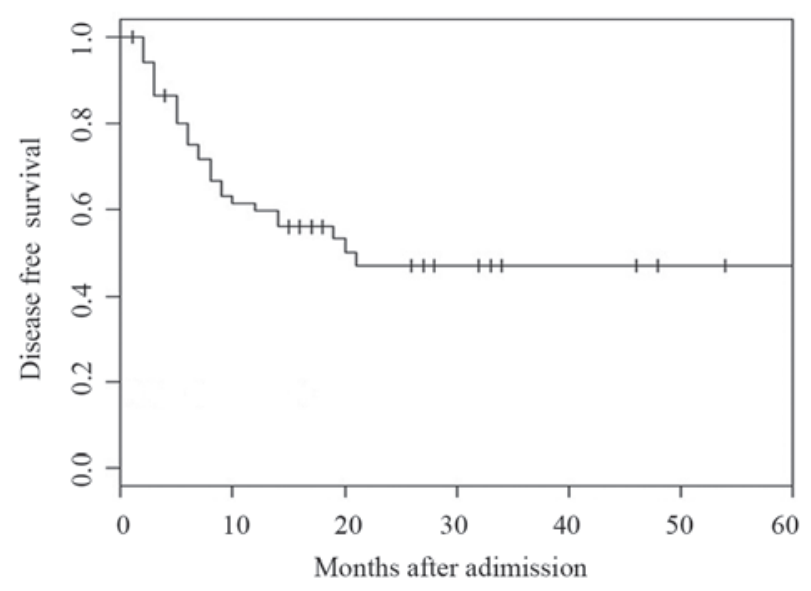

Figure 4. Disease free survival for the complete remission group. For patients exhibiting complete remission following the mitoxantrone and cytarabine regimen, the disease-free survival rate at 1,3 and 5 years was $59.7,47.1$ and $43 \%$, respectively.

treated with chemotherapy alone, and they were 31 and 17 , and 23 and 12 months, respectively $(\mathrm{P}=0.32$ and $\mathrm{P}=0.18)$.

Toxicity associated with the MAC regimen. Hematological toxicity and infections were the most prominent toxicities of the MAC treatment. All patients developed grade IV neutropenia, according to the NCI CTC4.0 criteria following the MAC therapy. The median duration of neutrophils $<0.5 \times 10^{9} / 1$ and platelets $<20 \times 10^{9} / 1$ was 11 (range, 6-22) days and 9 (range, 5-25) days, respectively. The patients received a median of 10 units of packed red cells (range, 3-29) and 6 units (range, 2-12) of platelets. The median duration of hospitalization was 26 (range, 19-56) days. Severe infections (grade III or IV) were observed in $29 \%(\mathrm{n}=26)$ of the cases, among which fungal was observed in $8 \%(n=7)$. The median duration of antibiotic therapy was 11 days. A total of $5(5.5 \%)$ patients had lightly elevated levels of aminotransferase and nausea, which resolved shortly following the completion of chemotherapy. Liver, kidney, circulatory failure or neuropathy following the MAC regimen was not observed.

\section{Discussion}

The outcomes of patients who experienced relapse are very poor despite age, cytogenetics and refractory free survival following CR1 (period from first CR until refractory or mortality) (15). For the majority of relapsed patients, fludarabine is used, as it acts synergistically with cytarabine. Certain regimens, including FLAG, Flag-Ida and fludarabine $\left(30 \mathrm{mg} / \mathrm{m}^{2} ; 0.5 \mathrm{~h} ; 3\right.$ consecutive days), cytarabine ( $1 \mathrm{~g} / \mathrm{m}^{2} ; 4 \mathrm{~h}$ infusions; 3 consecutive days), mitoxantrone (10 mg/m²; 3 consecutive days) and G-CSF (300 $\mu \mathrm{g}$ ) administered 12 hours prior to fludarabine, have been involved in studies for treating relapsed AML and in those with a poor-risk disease, with a CR rate ranging between 30 and $60 \%$. As an extensively used second-line regimen, purine nucleoside analogues, were combined with Ara-C for AML as salvage regimens in the last decade. The novel agent, cladribine, has been extensively employed in refractory/relapsed (RR)-AML. According to Pricea et al (16), the CR rate was $37.9 \%$ for cladribine $\left(5 \mathrm{mg} / \mathrm{m}^{2} ; 2 \mathrm{~h}\right.$ infusions; 5 consecutive days), cytarabine ( $2 \mathrm{~g} / \mathrm{m}^{2} ; 4 \mathrm{~h}$ infusions; 5 consecutive days) and G-CSF (300 $\mu \mathrm{g} ; 6$ days; CLAG) and the median OS was 7.3 months. In the initial relapse, the CR rate was $36.8 \%$ and median OS was 6.7 months. The Poland adult leukemia group (17) also reported that in poor risk refractory/relapsed patients with AML, 58\% achieved CR following one or two courses of CLAG with mitoxantrone (10 mg/m² 3 days), and the probability of OS at 4 years was $14 \%$. The probability of 4 year DFS was $30 \%$ for all 66 patients in CR. Therefore, a modified therapeutic regimen and combined use of chemotherapeutic agents for experienced primary induction failure or relapsed patients with AML is urgently required.

As a conventional cytotoxic agent, mitozantrone has no cross-resistance with other anthracycline agents and has significant potentially synergistic effects with cytarabine. With regards to the mechanism of action, mitozantrone is known to be active against leukemia cell lines resistant to daunorubicin (18). Liso et al and Kern et al $(9,10)$ reported a high-dose of cytarabine, combined with mitozantrone and etoposide, as a salvage regimen, and the CR rates were 47.4 and $47 \%$, the median OS rates were 8.1 and 4.2 months, respectively. The present study attempted to use cyclophosphamide instead of etoposide and comprise a novel regimen, MAC, as the salvage treatment. Cyclophosphamide, which was seldom used in the salvage regimens for AML, combined with high-dose cytarabine has represented a promising therapeutic approach to induce a second CR in younger patients (19). In the present study, cytarabine was performed with an sd in the MAC regimen to avoid severe marrow suppression. At the same time, the overall treatment costs were reduced. The CR rate $(74.7 \%)$ was encouraging. FLT3 (ITD/TKD) was the only statistically significant factor affecting $\mathrm{CR}$, while different cytogenetic risk groups revealed no influence on the treatment results. The result was similar to the observation made by others (20-21). The prognosis of adult patients with AML resistant to first-line or relapsed following CR1 within $<6$ months duration is poor. A striking a balance between the treatment-associated mortality, response rates of salvage regimens, and the likelihood of long-term overall survival are critical in planning a treatment approach for the patients with relapsed or experienced primary induction failure AML. High efficacy and a low toxicity profile 
are preferable properties of this regimen. Only once case of early mortality was observed. Severe infections (grade III or IV) were observed in $29 \%$ of the cases and they were all under control. The observed CR rate $(74.7 \%)$ can be considered excellent in this subset of patients, which included those with primary induction failure disease following standard or intermediate-dose Ara-c induction $(72.7 \% \mathrm{CR})$, even if the best response rate was obtained in the group of relapsed patients. As for relapsed patients, the response rate to salvage therapy correlated with the duration of the initial CR. Indeed, when the patients were stratified according to the initial CR duration into two groups ( $<6$ and $>6$ months), an improved outcome was observed for patients with initial CR duration $>6$ months, and the CR rate was 65.0 and $85.2 \%$, respectively. The median duration of OS and DFS was 22 and 10 months for the entire cohort. For patients who achieved CR following the MAC regimen, the DFS rate was 47.1 and $43 \%$, and the OS rate was 42.9 and $36.7 \%$ at 3 and 5 years, respectively. For patients who achieved CR or not, 1 and 3 year OS rate were 83.3, vs. $32.7 \%(\mathrm{P}<0.0001)$ and 57.3 , vs. $10.3 \%(\mathrm{P}<0.0001)$, respectively.

In conclusion, the MAC regimen may be a highly-effective salvage regimen in the treatment of AML and is worthy of further studies. The MAC regimen proved to be highly effective, as indicated by an overall CR rate of $74.7 \%$ and 5 years, and an OS and DFS of 36.7 and 43\%, respectively.

\section{Acknowledgements}

The authors would like to thank Professor Jacob M. Rowe (Rambam Medical Center, Haifa, Israel) for his constructive suggestions for preparing the manuscript.

\section{References}

1. Litzow MR: Progress and strategies for patients with relapsed and refractory acute myeloid leukemia. Curr Opin Hematol 14 130-137, 2007.

2. Cassileth PA, Harrington DP, Appelbaum FR, Lazarus HM, Rowe JM, Paietta E, Willman C, Hurd DD, Bennett JM, Blume KG, et al: Chemotherapy compared with autologous or allogeneic bone marrow transplantation in the management of acute myeloid leukemia in first remission. N Engl J Med 339: 1649-1656, 1998

3. Clavio M, Carrara P, Miglino M, Pierri I, Canepa L, Balleari E, Gatti AM, Cerri R, Celesti L, Vallebella E, et al: High efficacy of fludarabine-containing therapy (FLAG-FLANG) in poor risk acute myeloid leukemia. Haematologica 81: 513-520, 1996.

4. Estey EH: Treatment of relapsed and refractory acute myelogenous leukemia. Leukemia 14: 476-479, 2000.

5. Montillo M, Mirto S, Petti MC, Latagliata R, Magrin S, Pinto A, Zagonel V, Mele G, Tedeschi A and Ferrara F: Fludarabine, cytarabine and G-CSF (FLAG) for the treatment of poor risk acute myeloid leukemia. Am J Hematol 58: 105-109, 1998.

6. Byrne JL, Dasgupta E, Pallis M, Turzanski J, Forman K, Mitchell D, Haynes AP and Russell NH: Early allogeneic transplantation for refractory or relapsed acute leukaemia following remission induction with FLAG. Leukemia 13: 786-791, 1999.

7. Carella AM, Cascavilla N, Greco MM, Melillo L, Sajeva MR, Ladogana S, D'Arena G, Perla G and Carotenuto M: Treatment of 'poor risk' acute myeloid leukemia with fludarabine, cytarabine and G-CSF (FLAG regimen): A single center study. Leuk Lymphoma 40: 295-303, 2001.

8. Jackson G, Taylor P, Smith GM, Marcus R, Smith A, Chu P, Littlewood TJ, Duncombe A, Hutchinson M, Mehta AB, et al: A multicentre, open, non-comparative phase II study of a combination of fludarabine phosphate, cytarabine and granulocyte colony-stimulating factor in relapsed and refractory acute myeloid leukaemia and de novo refractory anaemia with excess of blasts in transformation. Br J Haematol 112: 127-137, 2001.
9. Liso V, lacopino P, Avvisati G,Petti MC,Broccia G,Carotenuto M, Falda M, Fazi P, Lazzarino M, Leoni P, et al: Outcome of patients with acute myeloid leukemia who failed to respond to a single course of first-line induction therapy: A GIMEMA study of 218 unselected consecutive patients. Gruppo italiano malattie ematologiche maligne dell'adulto. Leukemia 10: 1443-1452, 1996.

10. Kern W, Aul C, Maschmeyer G, Schönrock-Nabulsi R, Ludwig WD, Bartholomäus A, Bettelheim P, Wormann B, Büchner T and Hiddemann W: Superiority of high-dose over intermediate-dose cytosine arabinoside in the treatment of patients with high-risk acute myeloid leukemia: Results of a age-ajusted prospective randomized comparison. Leukemia 12: 1049-1055, 1998.

11. Bennet JM, Catovsky D, Daniel MT, Flandrin G, Galton DA, Gralnick HR and Sultan C: Criteria for the diagnosis of acute leukemia of megakaryocytic lineage (M7): A report of the French-American-British (FAB) cooperative group. Ann Intern Med 103: 460-462, 1985.

12. Vardiman JW, Harris NL and Brunning RD: The world health organization (WHO) classification of the myeloid neoplasms. Blood 100: 2292-2302, 2002

13. Slovak ML, Kopecky KJ, Cassileth PA, Harrington DH, Theil KS, Mohamed A, Paietta E, Willman CL, Head DR, Rowe JM, et al: Karyotypic analysis predicts outcome of pre-remission and post-remission therapy in adult acute myeloid leukemia: A southwest oncology group/eastern cooperative oncology group study. Blood 96: 4075-4083, 2000.

14. Cheson BD, Bennett JM, Kopecky KJ, Büchner T, Willman CL, Estey EH, Schiffer CA, Doehner H, Tallman MS, Lister TA, et al: Revised recommendations of the international working group for diagnosis, standardization of response criteria, treatment outcomes and reporting standards for therapeutic trials in acute myeloid leukemia. J Clin Oncol 21: 4642-4649, 2003.

15. Burnett AK, Wheatley K, Goldstone AH, Stevens RF, Hann IM, Rees JH and Harrison G; Medical Research Council Adult and Paediatric Working Parties: The value of allogeneic bone marrow transplant in patients with acute myeloid leukaemia at differing risk of relapse: Results of the UK MRC AML 10 trial. Br J Haematol 118: 385-400, 2002

16. Pricea SL, Lancetb JE, Georgea TJ, Wetzstein GA, List AF, Ho VQ, Fernandez HF, Pinilla-Ibarz J, Kharfan-Dabaja MA and Komrokji RS: Salvage chemotherapy regimens for acute myeloid leukemia: Is one better? Efficacy comparison between CLAG and MEC regimens. Leuk Res 35: 301-304, 2011.

17. Wierzbowska1 A, Robak1 T, Pluta1 A, Wawrzyniak E, Cebula B, Hołowiecki J, Kyrcz-Krzemień S, Grosicki S, Giebel S, Skotnicki AB, et al: Cladribine combined with high doses of arabinoside cytosine, mitoxantrone and G-CSF (CLAG-M) is a highly effective salvage regimen in patients with refractory and relapsed acute myeloid leukemia of the poor risk: A final report of the polish adult leukemia group. Euro J Haematol 80: 115-126, 2008.

18. Fujimoto $\mathrm{S}$ and Ogawa M: Antitumor activity of mitozantrone against murine experimental tumors: Comparative analysis against various antitumor antibiotics. Cancer Chemother Pharmacol 8: 157-162, 1982.

19. Schnetzke U, Fix P, Spies-Weisshart B, Schrenk K, Glaser A, Fricke HJ, LaRosée P, Hochhaus A and Scholl S: Efficacy and feasibility of cyclophosphamide combined with intermediate-dose or high-dose cytarabine for relapsed and refractory acute myeloid leukemia (AML). J Cancer Res Clin Oncol 140: 1391-1397, 2014.

20. De la Rubia J, Regadera AA, Martín G, Cervera J, Sanz G, Martínez J, Jarque I, García I, Andreu R, Moscardó F, et al: FLAG-IDA regimen (fludarabine, cytarabine, idarubicin and G-CSF) in the treatment of patients with high-risk myeloid malignancies. Leuk Res 26: 725-730, 2002.

21. Steinmetz HT, Schulz A, Staib P, Scheid C, Glasmacher A, Neufang A, Franklin J, Tesch H, Diehl V and Dias Wickramanayake P: Phase-II trial of idarubicin, fludarabine, cytosine arabinoside and filgrastim (Ida-FLAG) for treatment of refractory, relapsed and secondary AML. Ann Hematol 78: 418-425, 1999. 